\title{
Mycoplasma pathogenicity for humans and animals: an effort of complete genome sequencing and gene family analysis of Mycoplasma synoviae
}

\author{
Xu Jing Yi ${ }^{1}$, Atif Rehman ${ }^{3, *}$, Urooj Shahid ${ }^{4}$, Chen XiuHong ${ }^{1}$, Ashgar Abbas ${ }^{3}$, Khasif Hussain ${ }^{3}$, \\ Muhammad Asif Raza ${ }^{3}$, Hafiz Muhammad Ishaq ${ }^{3}$, He Sheng $\mathrm{Hu}^{1,2, *}$ and ${\mathrm{Guo} \mathrm{Li}^{2}}^{2}$ \\ ${ }^{1}$ Department of Veterinary Science, College of Agriculture, Ningxia University, Yinchuan 750000, China; ${ }^{2}$ Ningxia \\ Xiaoming Agriculture and Animal Husbandry Co. Ltd, Yinchuan 750000, China; ${ }^{3}$ Department of Veterinary and Animal \\ Sciences, Muhammad Nawaz Sharif University of Agriculture Multan, Pakistan; ${ }^{4}$ Government General Hospital GM Abad, \\ Faisalabad \\ *Corresponding author's e-mail: atif1632@hotmail.com; 617715465@qq.com
}

\begin{abstract}
Like humans, chickens can become sick from bacterial infections. Mycoplasma is a very small, slow-growing bacterium. There are multiple types of Mycoplasma that affect different animals, humans, and even plants and insects. In the current study, the whole genome of Mycoplasma synoviae was sequenced through the joint sequencing method of the third-generation PacBio and second-generation Illumina of the high-throughput sequencing platform, and the complete genome map of the Mycoplasma synoviae was successfully obtained. SMRT sequencing and assembly of whole genome: Canu v1.5/ WTDBG v2.2 software was used for preliminary assembly of filtered subreads. Pilon V1.22 software was used to further correct the assembled genome with high-quality second-generation data, and the genome with higher accuracy was finally obtained. Sequencing results showed that the Mycoplasma was composed of a $0.83 \mathrm{Mb}$ chromosome with a $\mathrm{G}+\mathrm{C}$ percentage of $28.42 \%$. Bioinformatics was used to predict the encoding protein genes, and it was found that Mycoplasma synoviae genome encoded a total of 1415 ORF open reading frames, including $1.73 \%$ of the repeats, 7 ribosomal RNAs, 34 transport RNAs, 4 non-coding RNAs and 12 pseudogenes. Mycoplasma synoviae genomes also contain 2 CRISPR, 1 gene island and 2 prophages. Using multiple strains of Mycoplasma gallisepticum, Mycoplasma iowae and Mycoplasma anatis as reference genomes, we constructed phylogenetic trees of 12 species of Mycoplasma. The genetic and taxonomic status of Mycoplasma synoviae strains were evaluated. The results showed that the sequencing strains of Mycoplasma synoviae and the model strain Mycoplasma synoviae WVU1853 were closely clustered together, suggesting the genetic relationship was relatively close and the sequencing strains were relatively conservative, and represent that they share the same genetic origin.

Keyword: Mycoplasma synoviae, genetic analysis, Sequencing, DNA, RNA.
\end{abstract}

\section{INTRODUCTION}

The discovery of double helix structure model of DNA molecule has encouraged the life science researcher four further discoveries on molecular level (Johansson and Pettersson, 2002). In 1975, Sanger and his co-worker made millions of identical copies of a DNA template by cloning or polymerase chain reaction (PCR), and completed the genome sequencing of bacteriophage X174, with a total length of 5375 bases, that has successfully opened the era of first-generation sequencing.

First generation sequencing usually refers to the chain termination method or chain degradation method, whose core is deoxyribonucleotide triphosphate (dNTPs) which interrupts the DNA synthesis reaction. The first human genome sketch was completed by using the first-generation sequencing technology (Feberwee et al., 2009). The generation of first-generation sequencing has greatly promoted the research of modern molecular biology (Kang et al., 2002). The sequencing read length of first-generation sequencing (Sanger sequencing) reached at $1000 \mathrm{bp}$, but its low throughput and high cost, Sanger sequencing is used for single sequence determination (Morrow et al., 1998). To overcome these deficiencies, researchers carried out working on sequencing technology, and now the second-generation sequencing technology has emerged at the historic moment. In the second-generation sequencing technology, the DNA samples to be tested is divided into small sequence fragments of 200-500bp by ultrasonic wave, and the DNA is amplified by bridging PCR on the surface of the sequencing instrument

Yi, X.J., A. Rehman, U. Shahid, C.X. Hong, A. Abbas, K. Hussain, M.A. Raza, H.M. Ishaq, H.S. Hu and G. Li. 2021. Mycoplasma pathogenicity for humans and animals: an effort of complete genome sequencing and gene family analysis of Mycoplasma synoviae. Pak. J. Agri. Sci.58:1379-1385.

[Received 16 Jun 2021; Accepted 19 Sep 2021; Published (online) 21 Sep 2021] 
using the coupling method (Jones et al., 2006). Finally, the optical signal is transformed into the sequencing base recognition by using the method of synthesis while sequencing, and the determination of specific sequences is completed (Shahid et al., 2013). Second-generation sequencing technologies include Roche's 454 technology, Illumina's Solexa, Hiseq technology and ABI's Solid technology, however, second-generation sequencing technology is still the mainstream technology in the field of life science. Second-generation sequencing quickly completes the determination of gene sequences, and transforming scientific research from a single local gene to gene fragment study of the entire genome, which is widely being used in the de novo sequencing and transcriptome sequencing of genomes (Zhu et al., 2018). Second generation sequencing technologies produce a massively parallel analysis with a high throughput and can sequence millions to billions of reads in a single run and the time required to generate the GigaBase sized reads is only a few days or hours making it best than the first-generation sequencing such as Sanger sequencing (Noormohammadi et al., 2003).

Yet the second-generation sequencing technology had several flaws limiting the research on DNA single base molecules, that are overcome by sequencing third-generation sequencing technology (Lockaby et al., 1998). Whole genome sequencing is the method to obtain the genome map of a species through de-novo sequencing of non-parametric species, stitching's and assembly by means of bioinformatics. High-quality reference genome is the basis for modern molecular biology to deeply understand the genetic composition and evolutionary traceback of species (Green and Sambrook, 2016; Green and Sambrook, 2017). Mycoplasma is often used as a model for the study of minimal life, which has the smallest genome among known prokaryotes. In the late 20th century, researchers completed the entire genome assembly of Mycoplasma genitalium G37 by shotgun sequencing, which was the first Mycoplasma to obtain a reference genome (Andrews, 2010). Papazisiet al. (2003), in 2003 performed whole genome sequencing on R strain of Mycoplasma gallisepticum (AE015450). Vasconcelos et al. (2005), in 2005, completed the assembly of Mycoplasma synoviae of Brazil 53 strains (AE017245) (Zerbino and Birney, 2008). Second-generation and thirdgeneration sequencing technology have brought the revolution in the genomics of Mycoplasma rapidly. 'By using these technologies, Mycoplasma synoviae, Mycoplasma gallisepticum and Mycoplasma iowae strains of poultry Mycoplasma had completed genome sequencing assembly'. To date, by using these technologies, Mycoplasma strain has reached more than 50 strains. However, further research is required to explore the further strain of Mycoplasma. Therefore, current study was conducted to analyses complete genome sequencing of Mycoplasma synoviae.

\section{MATERIALS AND METHODS}

Feeding and management: Birds were fed on the standard feed as described in the literature (Hussain et al., 2018; Xu et al., 2019; Arshad et al., 2020; Bajwa et al., 2020; Hussain et al., 2020; Kamran et al., 2020; Shahid et al., 2020; Sharif et al., 2020; Shahid et al., 2021). Birds were managed according to standard procedure as described in literature (Hussain et al., 2018; Xu et al., 2019). Birds were free from hunger and thirst and manged according to standard protocol of animal behavior and welfare (Muhammad et al., 2016; Aziz ur Rahman et al., 2017; Aziz ur Rahman et al., 2019; Muhmmad et al., 2020).

The source of Mycoplasma synoviae: The Ningxia University laboratory had already identified and preserved the Ningxia G-1 strain

Media and culture conditions: Mycoplasma synoviae special medium

Main instrument of the experiment and reagents: Instrument and reagents used in the current experiment were Ultra clean table, Supplement instrument manufacturer, Biochemical incubator, Micro tissue grinding machine, Ultramicro UV spectrophotometer (Li et al., 2014; Niu et al., 2017; He et al., 2018; Xia et al., 2018a; Xia et al., 2018b; Li et al., 2019; Qiu et al., 2019a; Qiu et al., 2019b; Qiu et al., 2020), Fluorescence real-time quantitative PCR instrument (Qiu et al., 2018; Rehman et al., 2019; Chen et al., 2020), Electrophoresis instrument, Sequencing instrument (Li et al., 2019; Qiu et al., 2019a; Qiu et al., 2020), Pacific Biosciences. Genomic DNA Extraction Kit; Accel-NGS ${ }^{\circledR} \cdot \mathrm{XL}$; Qubit ${ }^{\mathrm{TM}}$

Experimental method: Culture of strain and whole genome sequence

According to Mycoplasma synoviae strain amplification culture conditions, liquid culture, authenticate, DNA gene extraction by Mycoplasma synoviae was used as a template. Complete genome sequencing and assembly of Mycoplasma synoviae were performed based on PacBio third-generation sequencing platform. The main processes include

Extraction and purification of whole genome DNA and genomic DNA fragmentation: In this study, the Genomic DNA of Mycoplasma synoviae was extracted using Qiagen genomic-tip kit, and the whole process was carried out according to Protocol procedures. The Accel-NGS XL kit was used to prepare the genomic DNA library with the inserted fragment length of about $20 \mathrm{~Kb}$, which mainly included DNA fragmentation, fragment repair, addition of adapters, purification of DNA and fragment selection.

Construction of third-generation sequencing genomic DNA library: $10-15 \mu \mathrm{g}$ of high-quality whole genome DNA-SMRT Bell library was sent to Beijing Biometrics for sequencing. The sequencing platform relied on the third-generation singlemolecule real-time sequencing machine, PacBio, and the library size was $20 \mathrm{~Kb}$.Primers for full sequence of vlhA gene are presented in table 1. Raw data from the sequencing 
machine included DNA adapters and low-quality data. Quality control of sequencing data was completed by further filtering adapters and short reads to obtain total data sets (subreads). The number of subreads, the total number of bases of subreads, the value of subreadSN50, the average number of bases and the distribution of reads length were counted respectively to ensure the authenticity and reliability of the sequencing results. The details of PCR reaction system and conditions are presented in table 2 and 3 , respectively.

Table 1. Primers for full sequence of vlhA gene.

\begin{tabular}{llll}
\hline NAME & Primer & Sequence & Length \\
\hline vlhA & vlhA-f & 5'-GCCATTGCTCCTGCTGTTATA-3' & 773bp \\
& vlhA-r & 5'-GGGTAGTCCACTCGCATT-3' & \\
\hline
\end{tabular}

Table 2. PCR reaction system

\begin{tabular}{ll}
\hline Reagent & Volume \\
\hline Dd $\mathrm{H}_{2} \mathrm{O}$ & Up to $50 \mathrm{ul}$ \\
$2 \times \mathrm{Phanta}_{\text {Max Master Mix }}$ & $25 \mathrm{ul}$ \\
forward primer & $2 \mathrm{ul}$ \\
reverse primer & $2 \mathrm{ul}$ \\
vlhA Form & $2 \mathrm{ul}$ \\
total volume & $50 \mathrm{ul}$ \\
\hline
\end{tabular}

Table 3. PCR reaction conditions.

\begin{tabular}{lcll}
\hline Step & Temperature & Time & circulation \\
\hline predegeneration & $95^{\circ} \mathrm{C}$ & $4 \mathrm{~min}$ & \\
denaturation & $95^{\circ} \mathrm{C}$ & $40 \mathrm{sec}$ & \\
anneal & $56^{\circ} \mathrm{C}$ & $40 \mathrm{sec}$ & \\
extend & $72^{\circ} \mathrm{C}$ & $60 \mathrm{sec}$ & \\
Completely stretch & $72^{\circ} \mathrm{C}$ & $10 \mathrm{~min}$ & \\
save & $04^{\circ} \mathrm{C}$ & & \\
\hline
\end{tabular}

The assembled contig sequence was compared with the NT database, and finally assembled into a circular genome, that is, to complete the 0GAP genome completion map sequence. The software Circos was used to draw the genomic circle map, showing the relationship between genome components or locations more clearly.

\section{Whole genome analysis}

Genomic component analysis: Repeated sequences in Mycoplasma genome were predicted by REPEATMASKER V4.0.5 software. Prodiga V2.50 software was used to predict the coding genes of the assembled genome. The software trnascan-se v1.3.1 was used to predict the tRNA in the genome, and the software Infernal V1.1 was used to predict the rRNA in the genome and other ncRNAs besides tRNA and rRNA based on the RFAM v12.0 database. Bioinformatics analysis software Genblasta v1.0.1 was used to predict the protein sequence and carry out sequence alignment with the proteins of the same family in the Swiss-Prot public database, so as to retrieve the gene sequences homologous with the predicted genes on the genome. Bioinformatics software Genewise Wise2-2-0 was further used to mine the immature stop codon and frameshift mutation in the gene sequence to obtain pseudogenes.
Functional annotation of genome: GO database was used for gene functions and analysis.

\section{RESULTS AND DISCUSSION}

Results of genomic DNA extraction are presented in Fig. 1. The general features of the Mycoplasma synoviae genomes are listed in Table 4.

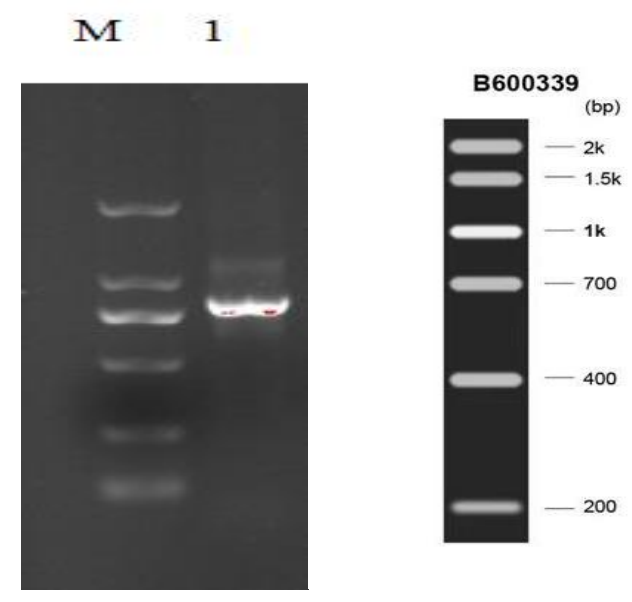

Figure 1. PCR amplification (M: Marker, 1:vlhA)

A total of $1.4 \mathrm{~GB}$ of filtered high-quality sequencing data were obtained by smart-seq technology, including 153,137 subreads, and the mean subreads length after filtering was 9,109bp. The length of N50 (SubreadSN50) was 11,656bp. After assembling the filtered subreads with CANU software, a contig with a size of $826,450 \mathrm{bp}$ was obtained, which completely covered the Mycoplasma synoviae genome. The GC Content of Mycoplasma synoviae genome was $28.42 \%$. Through multiple annotation and prediction, Mycoplasma synoviae genome contains a total of 1,415 protein coding genes, the total predicted gene length was532,710bp, accounting for $64.46 \%$ of the whole genome, 7 ribosomal RNA (rRNA), 34 transport RNA (tRNA), Four other noncoding RNAs (ncRNAs) and 12 pseudogene genes were identified.

Gene islands can be related to a variety of biological functions and can be divided into different subclasses based on their different functions. Mycoplasma synoviae genome contains a gene island with a length of 3,402bp and a total of 10 proteincoding genes. The presence of Prophage sequences may enhance the host's adaptability to the environment. The predicted results showed that Mycoplasma synoviae genome contained two prophages, with the length of 39,667bp and 11,430bp, respectively. As expected, the Mycoplasma synoviae mutation in the obg gene (Shahid et al., 2013), previously reported as a marker of the vaccine strain, was identified. 


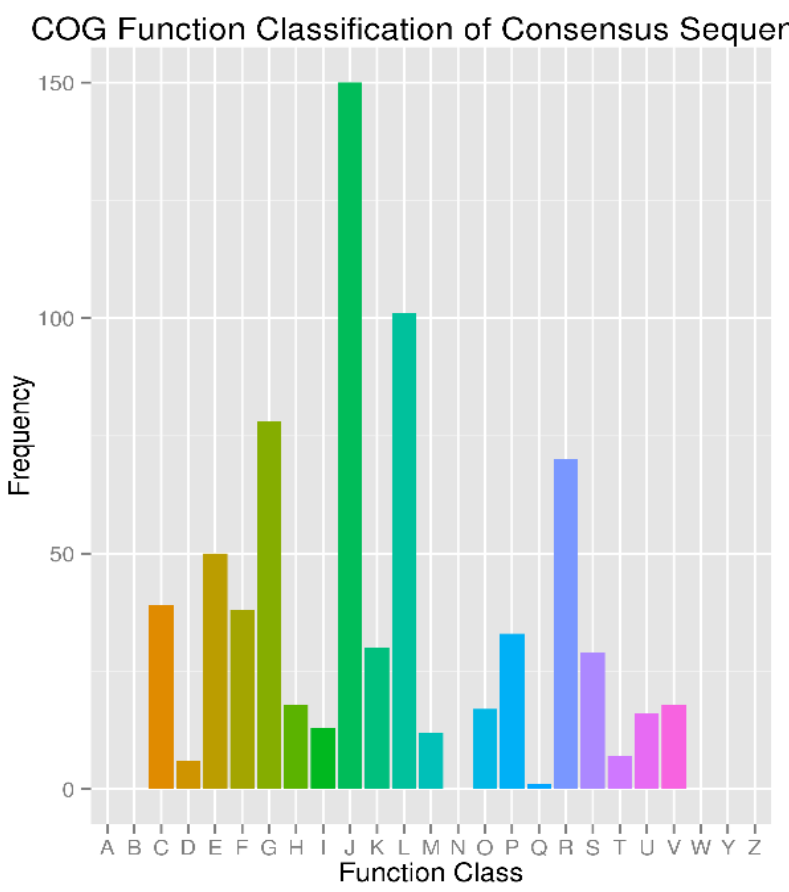

Figure 2. COG database function notes

Table 4. Genome statistics.

\begin{tabular}{lrr}
\hline Attribute & Number & Full/mean length (bp) \\
\hline Genome & 1 & 826,450 \\
Total genes & 1,415 & 532,710 \\
RNA genes & 45 & - \\
PesudoGene & 12 & 6,764 \\
CRISPR & 2 & 36 \\
Gene island & 1 & 3,402 \\
Prophage & 2 & 25,549 \\
\hline
\end{tabular}

COG is a database containing the Orthologous gene clusters of prokaryotes. Proteins that form each Orthologous group are assumed to be from an ancestor protein with similar functions. The potential function of prokaryotic genes can be annotated by BLAST comparison of the database. The results of COG annotation functional classification of Mycoplasma synoviae genomic data are shown in Fig. 2.

A total of 696 coding genes were annotated into 19 COG functional clusters. 150 genes were annotated into translation, ribosomal structure and biogenesis functional protein clusters, accounting for $20.66 \%$ of the total annotated genes. The basic breakdown of proteins into functional categories is similar to other mycoplasmas, with an emphasis on transport of compounds and protein synthesis (Westberg et al., 2004). As is typical of a newly sequenced genome, one-fourth to onethird of all proteins are without a well-annotated function. For genes that could be placed in clusters of COGs, the functional category was automatically determined. For genes that did not match to COGs, the gene was assigned to a COG category manually, with the majority of such cases being assigned to
A: RNA processing and modification [0 0\%]

B: Chromatin structure and dynamics [0 0\%]

C: Energy production and conversion [39 5.37\%]

D: Cell cycle control, cell division, chromosome partitioning [6 0.83\%]

E: Amino acid transport and metabolism [50 6.89\%]

F: Nucleotide transport and metabolism [38 5.23\%]

G: Carbohydrate transport and metabolism [78 10.74\%]

$\mathrm{H}$ : Coenzyme transport and metabolism [18 2.48\%]

I: Lipid transport and metabolism [13 1.79\%]

$\mathrm{J}$ : Translation, ribosomal structure and biogenesis [150 20.66\%]

K: Transcription [30 4.13\%]

L: Replication, recombination and repair [101 13.91\%]

M: Cell wall/membrane/envelope biogenesis [12 1.65\%]

$\mathrm{N}$ : Cell motility [0 0\%]

O: Posttranslational modification, protein turnover, chaperones [17 2.34\%]

P: Inorganic ion transport and metabolism [33 4.55\%]

Q: Secondary metabolites biosynthesis, transport and catabolism [1 0.14\%]

R: General function prediction only [70 9.64\%]

S: Function unknown [29 3.99\%]

T: Signal transduction mechanisms [7 0.96\%]

U: Intracellular trafficking, secretion, and vesicular transport [16 2.2\%]

V: Defense mechanisms [18 2.48\%]

W: Extracellular structures [0 0\%]

Y: Nuclear structure [0 0\%]

Z: Cytoskeleton [0 0\%]

the "Function Unknown" category. For genes with more than one COG category, only the first one (in alphabetical order) was used. Amino acid transport and metabolism, energy production and conversion, and energy production of carbohydrates and conversion), nucleotide transport and metabolism, replication, (recombination and repair) and general function prediction only, the number of genes identified by functional annotations exceeded $5 \%$. Only one gene was involved in secondary metabolites biosynthesis, transport and catabolism.

These results showed that the functions of most genes were still focused on supporting the growth and development of living organisms and energy metabolism, and the results of classification annotation were basically consistent with the commonness of mycoplasma. At the same time, 29 genes were annotated as functionally known genes by COG database, accounting for $3.99 \%$, and their functional potential is yet to be explored. The genome encodes numerous transporters with a wide range of substrates and possesses some additional transporters of unknown specificity. It appears that Mycoplasma synoviae should be able to transport and metabolize glucose, sucrose, fructose, maltose/maltodextrin, xylose, and trehalose as energy sources. This has been shown experimentally for glucose and sucrose (Jaffe et al., 2004). Glycerol should also be able to be used as an energy source, but no specific transporter for glycerol was found. The presence of mannose-6-phosphate isomerase (manA) suggests that mannose may also be metabolized, as is suggested elsewhere (Pollack, 2002). As is common to other mycoplasmas, Mycoplasma synoviae should 
be able to produce and use glycogen and starches. Fermentation of sugars appears to be the only method of ATP production in Mycoplasma synoviae. A complete glycolysis pathway is present that terminates in the formation of lactate. Most of the nonoxidative branch of the pentose phosphate pathway is present except for the reaction normally carried out by transaldolase. This is similar to the situation in Mycoplasma synoviae and other mycoplasmas, and presumably this function is carried out by an as-yetunrecognized protein (Pollack, 2002; Westberg et al., 2004). A variety of DNA restriction/modification system enzymes are detected in Mycoplasma synoviae. This may account for their recalcitrance to genetic manipulation via transposon mutagenesis (J. Jaffe, unpubl.; M. Miyata, pers. comm.) even though it is a popular mutagenesis technique in other mycoplasma species. A similar obstacle was overcome in Mycoplasma arthritidis by using an appropriate DNA modification enzyme to circumvent that species' endogenous restriction enzymes (Voelker and Dybvig, 1996). GO(Gene Ontology) database contains the annotation of target genes and their products, which is based on cellular component, molecular function and biological process.

The aim is to define and characterize the function of genes and proteins in multiple species. As shown in Figure 3, a total of 912 genes in Mycoplasma synoviae genome were annotated into GO database, among which 392 genes in cell components were annotated into cell-related terms. In the molecular function module, the number of genes related to catalytic activity and binding was the highest, 658 and 581, respectively. Annotation genes were mainly found in metabolic process and cellular process, which contained 595 and 534 genes respectively (Fig. 3).

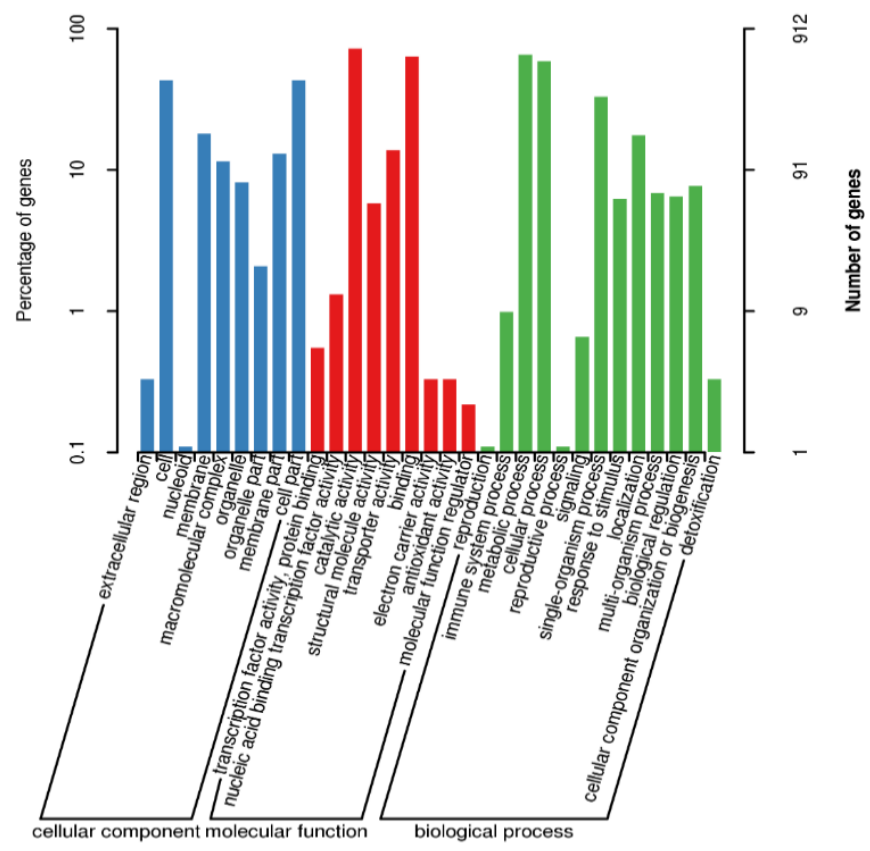

Figure 3. Go database function notes
The results showed that some items of biological process were involved in regulating cellular process, single-organism process and metabolic process; some items of cellular component were involved in regulating cell part, intracellular membrane-bounded organelle; and some items of molecular functions were involved in regulating protein binding, organic cyclic compound binding and catalytic activity as reported elsewhere (Zhao et al., 2020).

Conclusion: Based on results it is concluded that the sequencing strains of Mycoplasma synoviae and the model strain Mycoplasma synoviae WVU1853 were closely clustered together, suggesting the genetic relationship was relatively close and the sequencing strains were relatively conservative, and represent that they share the same genetic origin.

Acknowledgement: The first author sac knowledges the financial grant from Ningxia University (Ningxia Xiaoming Agriculture and Husbandry Co., Ltd.), Yinchuan, Ningxia, China

\section{REFERENCES}

Albright, R.A., K. Joh and J.H. Morais-abral. 2007. Probing the structure of the dimeric KtrB membrane protein. J. Biol. Chem. 282:35046-35055.

Andrews, S. 2010. Fast QC: A quality control tool for high throughput sequence data. Retrieved from http://www.bioinformatics.babraham.ac.uk/projects/fast qc.

Arshad, M., S. Bhatti, I. Hassan, M. Rahman and M. Rehman. 2020. Effects of bile acids and lipase supplementation in low-energy diets on growth performance, fat digestibility and meat quality in broiler chickens. Braz. J. Poult. Sci. 22: 1-8. doi:http://dx.doi.org/10.1590/1806-9061-20201258

Aziz ur Rahman, M., X. Chuanqi, S. Huawei and C. Binghai. 2017. Effects of hay grass level and its physical form (full length vs. chopped) on standing time, drinking time, and social behavior of calves. J. Vet. Behav. 21: 7-12. doi:10.1016/j.jveb.2017.06.004

Aziz ur Rahman, M., C. Xia, L. Ji, B. Cao and H. Su. 2019. Nutrient intake, feeding patterns, and abnormal behavior of growing bulls fed different concentrate levels and a single fiber source (corn stover silage). J. Vet. Behav. 33: 46-53. doi:10.1016/j.jveb.2019.03.003

Bajwa, M.H., M.A. Mirza, G. Ahmad, T. Mahmood and A.U.R. Muhammad. 2020. Comparative efficacy of vitamin $\mathrm{D}$ sources on growth response and bone mineralization in broilers. Pak. J. Agric. Sci. 57: 255-261.

Chen, D., J. Yan, W. Shen, Y. Song, X. Lan, K. Yi and A.U.R. Muhammad. 2020. Effect of inclusion of HMBi in the ration of goats on feed intake, nutrient digestibility, 
rumen bacteria community and blood serum parameters. J. Anim. Physiol. Anim. Nutr. 104: 987-997.

Feberwee, A., J.J. DeWit and W.J.M. Landman. 2009. Induction of eggshell apex abnormalities by Mycoplasma synoviae: Field and experimental studies. Avian Pathol. 38:77-85.

Green, M.R. and J. Sambrook. 2016. Precipitation of DNA with ethanol. Cold Spring Harbor Protocols, 12:11161120 .

Green, M.R. and J. Sambrook. 2017. Isolation of highmolecular-weight DNA using organic solvents. Cold Spring Harbor Protocols. 4:356-359, pdb-prot093450.

He, Y., W. Niu, Q. Qiu, C. Xia, T. Shao, H. Wang, Q. Li, Z. $\mathrm{Yu}, \mathrm{Z}$. Gao, M.A.U. Rahman, H. Su and B. Cao. 2018. Effect of calcium salt of long-chain fatty acids and alfalfa supplementation on performance of Holstein bulls. Oncotarget 9: 3029-3042.

Hussain, M., A. Mahmud, J. Hussain, S. Qaisrani, S. Mehmood and A. Rehman. 2018. Subsequent effect of dietary lysine regimens fed in the starter phase on the growth performance, carcass traits and meat chemical composition of aseel chicken in the grower phase. Braz. J. Poult. Sci. 20:455-462.

Hussain, M., A. Mahmud, J. Hussain, S.N. Qaisrani, S. Mehmood, S. Ahmad and A.U. Rehman. 2020. Effect of dietary amino acid regimens on growth performance and bodyconformation and immune responses in Aseel chicken. Indian J. Anim. Res. 54:53-58.

Jaffe, J.D., M. Miyata and H.C. Berg. 2004. The energetics of gliding motility in Mycoplasma mobile. J. Bacteriol. 186:4254-4261.

Johansson, K.E. and B. Pettersson. 2002. Taxonomy of mollicutes. In S. Razin R. Herrmann (Eds.), Molecular Biology and Pathogenicity of fmycoplasmas. Boston: Springer. pp.1-132

Jones, J.F., K.G. Whithear, P.C. Scott, and A.H. Noormohammadi. 2006. Duration of immunity with Mycoplasma synoviae: Comparison of the live attenuated vaccine MS-H (Vaxsafe MS) with its wild-type parent strain, 86079/7NS. Avian Dis.50:228-231.

Kamran, J., S. Mehmood, M. Rahman, A. Mahmud, M. Hussain, A. Rehman, S. Khalil and S. Qamar. 2020. Effect of fat sources and emulsifier supplementation in broiler starter, grower and finisher diets on performance, nutrient digestibility, and carcass parameters. Braz. J. Poult. Sci. 22: 1-10.

Kang, M.S., P. Gazdzinski and S.H. Kleven. 2002. Virulence of recent isolates of Mycoplasma synoviae in turkeys. Avian Dis. 46:102-110.

Li, L., Y. He, M. Aziz-ur-Rahman and B. Cao. 2014. Effects of different dietary energy and rumen-degradable protein levels on rumen fermentation, nutrients apparent digestibility and blood biochemical constituents of chinese crossbred yellow bulls. Pak. Vet. J. 34: 367-371.
Li, L., M.A.U. Rahman, R. Li, J. Hu, Q. Wang and A. Zhang. 2019. Resistant starch type 4 affects colon morphology, fermentation and microflora in rats. Int. J. Agric. Biol. 22:665-668.

Lockaby, S.B., F.J. Hoerr, L.H. Lauerman and S.H. Kleven. 1998. Pathogenicity of Mycoplasma synoviae in broiler chickens. Vet. Pathol. 35:178-190.

Morrow, C.J., J.F. Markham and K.G. Whithear.1998. Production of temperature-sensitive clones of Mycoplasma synoviae for evaluation as live vaccines. Avian Dis. 42:667-670.

Muhammad, A.U., C.Q. Xia and B.H. Cao. 2016. Dietary forage concentration and particle size affect sorting, feeding behaviour, intake and growth of Chinese Holstein male calves. J. Anim. Physiol. Anim. Nutr. 100: 217-223. doi:10.1111/jpn.12349

Muhmmad, A.U.R., T. Zulqurnain, S. Muhammad, Y. Muhammad, M.R. Virk, M.A. Mirza, A. Fawwad and F. Naeem. 2020. Effect of lysozyme, tributyrin, Bacillus amyloliquefaciens SC06 and enramycin on growth performance, nutrient digestibility and carcass characteristics of broiler during finisher phase. Pak. J. Agric. Sci. 57: 949-956.

Niu, W., Y. He, C. Xia, M.A.U. Rahman, Q. Qiu, T. Shao, Y. Liang, L. Ji, H. Wang and B. Cao. 2017. Effects of replacing Leymus chinensis with whole-crop wheat hay on Holstein bull apparent digestibility, plasma parameters, rumen fermentation, and microbiota. Sci Rep 7: 2114. doi:10.1038/s41598-017-02258-2

Noormohammadi, A.H., J.F. Jones, K.E. Harrigan and K.G. Whithear. 2003. Evaluation of the non-temperaturesensitive field clonal isolates of the Mycoplasma synoviae vaccine strain MS-H. Avian Dis. 47:355-360.

Papazisi L., T.S. Gorton, G. Kutish, P.F. Markham, G.F. Browning, D.K. Nguyen, S. Swartzell, A. Madan, G. Mahairas and S.J. Geary. The complete genome sequence of the avian pathogen Mycoplasma gallisepticum strain R(low). Microbiology (Reading). 2003 Sep; 149:23072316.

Pollack, J.D. 2002. Central carbohydrate pathways: Metabolic flexibility and the extra role of some "housekeeping" enzymes. In Molecular biology and pathogenicity of mycoplasmas (eds. S. Razin and R. Herrmann),. Kluwer Academic/Plenum, New York.pp. 163-200

Qiu, Q., C. Gao, M. Aziz ur Rahman, B. Cao and H. Su. 2020. Digestive ability, physiological characteristics, and rumen bacterial community of holstein finishing steers in response to three nutrient density diets as fattening phases advanced. Microorganisms 8: 335.

Qiu, Q., C. Gao, Z. Gao, M.A.U. Rahman, Y. He, B. Cao and H. Su. 2019a. Temporal dynamics in rumen bacterial community composition of finishing steers during an 
adaptation period of three months. Microorganisms 7: 410.

Qiu, Q., T. Shao, Y. He, A.U. Muhammad, B. Cao and H. Su. 2018. Applying real-time quantitative PCR to diagnosis of freemartin in Holstein cattle by quantifying SRY gene: a comparison experiment. Peer J. 6:e4616.

Qiu, Q., Y. Zhu, X. Qiu, C. Gao, J. Wang, H. Wang, Y. He, M.A.U. Rahman, B. Cao and H. Su. 2019b. Dynamic variations in fecal bacterial community and fermentation profile of holstein steers in response to three stepwise density diets. Animals 9: 560.

Rehman, A., H. Hesheng, M.A.U. Rahman, X. Jingyi, L. Shuang, G. Yannan and S.H. Qamar. 2019. Evaluation of efficacy of compound chinese medicinal herbs against mycoplasma synoviae using different lab tests in mouse and chicken. Int. J. Agric. Biol. 22:647-654.

Sanger, F. and A. Coulson. 1975. A rapid method for determining sequences in DNA by primed synthesis with DNA polymerase. J. Mol. Biol. 94:441-448.

Shahid, M.A., P.F. Markham, J.F. Markham, M.S. Marenda and A.H. Noormohammadi. 2013. Mutations in GTP binding protein Obg of Mycoplasma synoviae vaccine strain MS H: Implications in temperature-sensitivity phenotype. PLoS One.8: e73954.

Shahid, I., M. Sharif, M. Yousaf, F. Ahmad, U. Anwar, A. Ali, M. Hussain and M. Rahman. 2020. Emulsifier Supplementation Response in Ross 308 Broilers at 1-10 Days. Braz. J. Poult. Sci. 22:1-6.

Shahid, I., M. Sharif, M. Yousaf, F. Ahmad, M. Virk, M. Bilal, U. Anwar, A. Ali, M. Hussain and M. Chishti. 2021. Effect of exogenous emulsifier (lysophospholipid) supplementation in the broiler diet, on the feed intake and growth performance during grower phase. Braz. J. Poult. Sci.23:1-8.

Sharif, M., A. Ali, U. Anwar, F.A. Chisti, S. Jameel, M.Q. Bilal, M. Hussain, F. Ahmad, M. Yousaf and A. Naveed. 2020. Effect of different levels of energy and lysine on the production performance of laying hens. Pak. J. Agric. Sci. 57: 1653-1659.

Vasconcelos AT., H.B. Ferreira, C.V. Bizarro, S.L. Bonatto, M.O. Carvalho, P.M. Pinto, D.F. Almeida, L.G. Almeida, R. Almeida, L. Alves-Filho and E.N. Assunção. 2005. Swine and poultry pathogens: The complete genome sequences of two strains of Mycoplasma hyopneumoniae and a strain of Mycoplasma synoviae. J. Bacteriol. 87:5568-5577.

Voelker, L.L. and K. Dybvig, 1996. Gene transfer in Mycoplasma arthritidis: Transformation, conjugal transfer of Tn916, and evidence for a restriction system recognizing AGCT. J. Bacteriol. 178:6078-6081.

Westberg, J., A. Persson, A. Holmberg, A. Goesmann, J. Lundeberg, K.E. Johansson, B. Pettersson and M. Uhlen. 2004. The genome sequence of Mycoplasma mycoides subsp. mycoides SC type strain PG1T, the causative agent of contagious bovine pleuropneumonia (CBPP). Genome Res. 14:221-227.

Xia, C., M. Aziz Ur Rahman, H. Yang, T. Shao, Q. Qiu, H. $\mathrm{Su}$ and B. Cao. 2018a. Effect of increased dietary crude protein levels on production performance, nitrogen utilisation, blood metabolites and ruminal fermentation of Holstein bulls. Asian-Australas Journal of Animal Science 31: 1643-1653.

Xia, C.Q., A.U.R. Muhammad, W. Niu, T. Shao, Q. Qiu, S. Huawei and B. Cao. 2018b. Effects of dietary forage to concentrate ratio and wildrye length on nutrient intake, digestibility, plasma metabolites, ruminal fermentation and fecal microflora of male Chinese Holstein calves. Journal of Integrative Agriculture 17: 415-427.

$\mathrm{Xu}$, J., H. Hesheng, R. Atif, Y. Guo and A.u.R. Muhammad. 2019. Effect of supplementation of chinese herbal extracts in drinking water on growth performance, nutrient utilization and immune response in broilers. Int. J. Agric. Biol. 22: 933-938.

Zhao, Y., Y. Fu, M. Zou, Y. Sun, X. Yin, L. Niu, Y. Gong and X. Peng. 2020. Analysis of deep sequencing exosomemicroRNA expression profile derived from CP-II reveals potential role of gga-miRNA-451 in inflammation. J. Cell. Mol. Med. 24:6178-6190.

Zhu, L., M.A. Shahid, J. Markham, G.F. Browning, A.H. Noormohammadi and M.S. Marenda. 2018. Genome analysis of Mycoplasma synoviae strain MS-H, the mostcommon $M$. synoviae strain with a worldwide distribution. BMC Genomics. 19:117.

Zerbino, D.R. and E. Birney. 2008. Velvet: Algorithms for de novo short read assembly using de Bruijn graphs. Genome Res.18:821-829. 